\title{
Phase Diagram of the Square-Lattice Three-State Potts Antiferromagnet with Staggered Polarization Field
}

\author{
Hiromi Otsuka and Yutaka Okabe \\ Department of Physics, Tokyo Metropolitan University, Tokyo 192-0397 Japan
}

(Dated: December 1, 2018)

\begin{abstract}
We study a square-lattice three-state Potts antiferromagnet with a staggered polarization field at finite temperature. Numerically treating the transfer matrices, we determine two phase boundaries separating the model-parameter space into three parts. We confirm that one of them belongs to the ferromagnetic three-state Potts criticality, which is in accord with a recent prediction, and another to the Ising type; these are both corresponding to the massless renormalization-group flows stemming from the Gaussian fixed points. We also discuss a field theory to describe the latter Ising transition.

PACS numbers: 05.50.+q, 05.70.Jk, 64.60.Fr
\end{abstract}

It is widely recognized that strong frustrations can provide a ground state with an extensive entropy and prevent long-range orderings of systems. The simplest example may be the triangular-lattice antiferromagnetic (AF) Ising model, whose ground state is critical and exhibits power-law decays of correlation functions 1, 2]. Although, unlike in the ferromagnetic $(\mathrm{F})$ cases, the models of this type may depend on their details (e.g., lattice structures), the field theories used to describe the ground state and lower-energy excitations have attracted much attention. Further, recent interests in this area are rather focused on mutual relations of fixed points (e.g., crossovers of criticalities) embedded in the renormalization-group (RG) flows 3], so the understandings of their universal properties are quite important [4].

The AF three-state $(q=3)$ Potts model on the square lattice $\Lambda$ exhibits the same properties as those systems; its Hamiltonian is described by using the ternary variables $\sigma_{j}=0,1,2(j \in \Lambda)$ as

$$
H_{0}=J \sum_{\langle j, k\rangle} \delta_{\sigma_{j}, \sigma_{k}} \quad(J>0),
$$

where the sum runs over all nearest-neighboring $(\mathrm{NN})$ pairs. While its ground-state 5, 6, 7, 8, 9, 10, 11] and finite temperature properties [12, 13] have been intensively investigated, a possibility of the crossover behavior from the AF to the $\mathrm{F}$ three-state Potts criticality has been recently proposed on the basis of a continuous field theory 14. In close relation to the classical spin system, the realization of the field theory was found in the 1D quantum spin system (i.e., a frustrated XXZ Heisenberg chain in magnetic fields), and it can show the $\mathrm{F}$ three-state Potts criticality in its ground state [15]. In this Letter, we quantitatively investigate the possibility of crossover by introducing a square-lattice three-state Potts model defined below, which is also relevant to 1D quantum systems. Before presenting the formulation of our investigation, we shall briefly refer to the relating research so far.

Since the ground state of $H_{0}$ is equivalent to the sixvertex model on the ice point, it shows the Gaussian criticality with the conformal anomaly number $c=1$ [5, 6, 7, 8, 9]. This sort of equivalence was used to determine the properties of lower-energy excitations: den Nijs, Nightingale, and Schick found that both the uniform $s_{j}=e^{2 \pi i \sigma_{j} / 3}$ and the staggered magnetizations $S_{j}=(-1)^{j} s_{j}$ are relevant with scaling dimensions $x_{s}=\frac{2}{3}$ and $x_{S}=\frac{1}{6}$, respectively [7. Here, $(-1)^{j}= \pm 1$ for $j$ in the even (odd) sublattice $\Lambda_{ \pm}$. It was pointed out that the scaling dimensions of relevant scalar operators can take three values, and other than above two, the staggered polarization $P_{j}=(-1)^{j} \sum_{k}^{\prime}\left(2 \delta_{\sigma_{j}, \sigma_{k}}-1\right)$ takes $x_{P}=\frac{3}{2}$ [the sum is over $k$ next-nearest neighboring (NNN) with j] [8].

Numerical studies have been also performed 10, 11]: Salas and Sokal using the Monte Carlo (MC) simulations at zero temperature succeeded in confirming the lowest two scaling dimensions, but the estimation for the third one exhibits a deviation due to its larger energy scale 11. The scaling dimensions can be also obtained by the transfer matrix technique; the lowest two were accurately obtained by de Queiroz, but the third could not be found [16]. More profound understanding at finite temperature was brought about by Cardy, Jackobsen, and Sokal [12]. They pointed out that there are two types of excitations controlled by the thermal scaling field $u=e^{-J / k_{\mathrm{B}} T}$, i.e., the relevant one with $x_{\epsilon}=\frac{3}{2}$, and the marginal one, and that both of these are necessary to explain the exotic corrections to scaling observed in the MC data 13]. Nevertheless, as its main role, the energy operator $\epsilon_{j}=\sum_{k} \delta_{\sigma_{j}, \sigma_{k}}$ (the sum is over $k \mathrm{NN}$ with $j$ ) brings about the second-order phase transition with the divergent correlation length $\xi \propto u^{-1 /\left(2-x_{\epsilon}\right)}$ 8, 12.

Recently, Delfino argued the "bosonization" descriptions of the transition and above-mentioned excitations 9, 14]. He also provided their discrete symmetry properties, and then concluded that the criticality in the ground state can exhibit the crossover behavior to the three-state Potts criticality with $c=\frac{4}{5}$ as a resultant of the competing relevant perturbations $\epsilon_{j}$ and $P_{j}$, and its description is given by the self-dual sine-Gordon model with the dimensionless coupling $\beta^{2}=6 \pi$ (see also Ref. [17). 
Based on these developments, especially for the aim of quantitative understandings on the competitions between relevant perturbations and the resultant crossovers of criticalities, we shall introduce the following model, namely, the square-lattice AF three-state Potts model with the staggered polarization field defined by the reduced Hamiltonian $\mathcal{H}(K, V)=H / k_{\mathrm{B}} T$ with

$$
\mathcal{H}(K, V)=K \sum_{\langle j, k\rangle} \delta_{\sigma_{j}, \sigma_{k}}-V \sum_{[j, k]}(-1)^{j} \delta_{\sigma_{j}, \sigma_{k}}
$$

The second sum runs over all NNN pairs $[j, k]$, so that the sublattice symmetry is explicitly broken for nonzero $V$, but the $\mathbf{S}_{3}$ symmetry associated with the global permutations of the ternary variables is preserved. Other than $(K, V)=(\infty, 0)$, there are two special points in this model: When $K=0$, the system decouples into those defined on $\Lambda_{ \pm}$, and thus $\left(0, V_{3}^{\text {ex }}\right)$ and $(0, \infty)$ correspond to the exact transition point of the $\mathrm{F}$ three-state Potts model on $\Lambda_{+}$and to the ground state of the AF threestate Potts model on $\Lambda_{-}$, respectively $\left[V_{q}^{\text {ex }}:=\ln (1+\sqrt{q})\right]$ [18]. Further, when $K>0$, we expect that the disordered phase at $V \ll 1$ will change into a phase with the $\mathrm{F}$ ordering on $\Lambda_{+}$and with the AF ordering on $\Lambda_{-}$satisfying the exclusion condition of states at $V \gg 1$. Therefore, we shall numerically clarify the phase diagram in the twodimensional model-parameter space.

Let us consider $\Lambda$ with $M$ rows of $L$ sites wrapped on a cylinder and take an even number $L$ and the limit $M \rightarrow \infty$. Then, the site $j \in \Lambda$ is specified by $l \in[1, L]$ and $m \in[1, M]$. For this system, we can define the transfer matrix $\mathbf{T}(L)$ connecting the NNN pair of rows, and denote its eigenvalues as $\left\{\lambda_{\alpha}(L)\right\}$ ( $\alpha$ specifies a level). The conformal field theory provides direct expressions for $c$ and $x_{\alpha}$ (the scaling dimensions) of critical systems by using the eigenvalues [19, 20]:

$$
\begin{aligned}
& -\zeta \ln \left[\lambda_{I}(L)\right] \simeq L f-\pi c / 6 L+b / L^{3}, \\
& -\zeta \ln \left[\lambda_{\alpha}(L)\right]+\zeta \ln \left[\lambda_{I}(L)\right] \simeq 2 \pi x_{\alpha} / L,
\end{aligned}
$$

where $\lambda_{I}$ is the largest one corresponding to the ground state. $\zeta, f$, and $b$ are the geometric factor $\left(\frac{1}{2}\right)$, a free energy per site, and a nonuniversal constant, respectively. Here, it should be noted that the discrete symmetries of the lattice Hamiltonian, e.g., the translation $\mathcal{T}$ (e.g., $\left.\sigma_{l, m} \rightarrow \sigma_{l+2, m}\right)$, the space inversion $\mathcal{P}$ (e.g., $\left.\sigma_{l, m} \rightarrow \sigma_{L-l+2, m}\right)$, and the $\mathbf{S}_{3}$ symmetry $\left[\sigma_{l, m} \rightarrow g\left(\sigma_{l, m}\right)\right.$ for $g \in \mathbf{S}_{3}$ ] are crucial not only for a reduction of computational efforts, but also for the proper specification of the level $\alpha$ 21]. This can be clearly demonstrated by evaluating the third scaling dimension $x_{P}$ at $(K, V)=(\infty, 0)$. According to Ref. 9], the staggered polarization operator is invariant for all $g \in \mathbf{S}_{3}$ and has the wave number $\pi / a$ so that the corresponding level $-\zeta \ln \left[\lambda_{P}(L)\right]$ should be found in the subspace specified by these symmetries. In Table \ we give the numerical data for the scaling
TABLE I: The size dependence of the scaling dimension $x_{P}$. The fitting $x_{P}(L)=x_{P}(\infty)+b_{1} / L^{2}+b_{2} / L^{4}$ is performed using the data of $L=10,12$, and 14 .

\begin{tabular}{cccccc}
\hline \hline & $L=8$ & 10 & 12 & 14 & $\infty$ \\
\hline$x_{P}(L)$ & 1.6801468 & 1.6065435 & 1.5710939 & 1.5510358 & 1.5007 \\
\hline \hline
\end{tabular}

dimension $x_{P}(L)$ estimated from Eq. (4). The extrapolated value does not deviate more than $0.1 \%$ from the theoretical one, $\frac{3}{2}$, and the level is not the third one in the whole space, so the characterization of the excitation levels is essentially important (for other exponents, see Ref. 16]).

Now, according to the prediction of the crossover, it is plausible that the critical RG flow starts out of $(K, V)=(\infty, 0)$ and arrives at the point $\left(0, V_{3}^{\mathrm{ex}}\right)$. Thus, at this stage, our numerical task is to determine the line $V_{3}(K)$. For this purpose, we shall employ the phenomenological RG (PRG) method 22]. Let us denote the left-hand side of Eq. (4) as $\Delta E_{\alpha}(K, V, L)$ (i.e., an excitation gap), then we shall numerically solve the following PRG equation for a given value of $K$ with respect to $V$ : $L \Delta E_{\alpha}(K, V, L)=L^{\prime} \Delta E_{\alpha}\left(K, V, L^{\prime}\right)$. Since this is satisfied by the gap $\Delta E_{\alpha}(K, V, L) \propto 1 / L$, the obtained value can be regarded as the size-dependent estimate of the transition point, say $V_{3}(K, \bar{L})$ [we take $\bar{L}=\left(L+L^{\prime}\right) / 2$ and $L^{\prime}=L-2$ in the following]. Further, there are two critical fixed points connected by the RG flow, so a relationship between lower-energy excitations on these fixed points, namely the ultraviolet-infrared (UV-IR) operator correspondence, is quite important for the choice of the excitation $\alpha$. Along the flow, the conjecture, $S_{j} \rightarrow \tilde{s}_{j}$ and $S_{j}^{*} \rightarrow \tilde{s}_{j}^{*}$, has been proposed [17]. $\tilde{s}_{j}$ is the magnetic operator on the IR fixed point with the three-state Potts criticality, whose scaling dimension is $x_{\tilde{s}}=\frac{2}{15}$. Since the excitation $\tilde{s}_{j}$ provides the lowest energy level, we shall focus our attention on $\Delta E_{\tilde{s}}(K, V, L)$ stemming from the level of $S_{j}$ on the UV Gaussian fixed point.

The exact diagonalization calculations of $\mathbf{T}(L)$ with $L=4-14$ are performed by using the Lanczos algorithm, where the discrete symmetries $\mathcal{T}, \mathcal{P}$, and $g \in \mathbf{S}_{3}$ are utilized. We plot examples of $L$ and $V$ dependences of the scaled gap $(L / 2 \pi) \Delta E_{\tilde{s}}(K, V, L)$ in Fig. 1] and find the crossing points. While non-trivial finite-size corrections may affect their behaviors, we shall extrapolate them to the thermodynamic limit according to the finite-size scaling argument 23] : Suppose a single-power formula, i.e., $V_{3}(K, L)-V_{3}(K) \propto L^{-\psi_{3}}$, then the exponent is given as $\psi_{3}=\omega_{3}+1 / \nu$, where $\omega_{3}$ and $\nu$ are the correction exponent and the critical exponent of the correlation length. For the $\mathrm{F}$ three-state Potts model, $\omega_{3}=\frac{4}{5}$ [24] and $1 / \nu=\frac{6}{5}$, so we shall use the formula with $\psi_{3}=2$ and extrapolate $V_{3}(K, \bar{L})$ to the limit.

For convenience, we define the reduced couplings $(u, v)=\left(e^{-K}, 1-e^{-V}\right)$ and compactify the parame- 


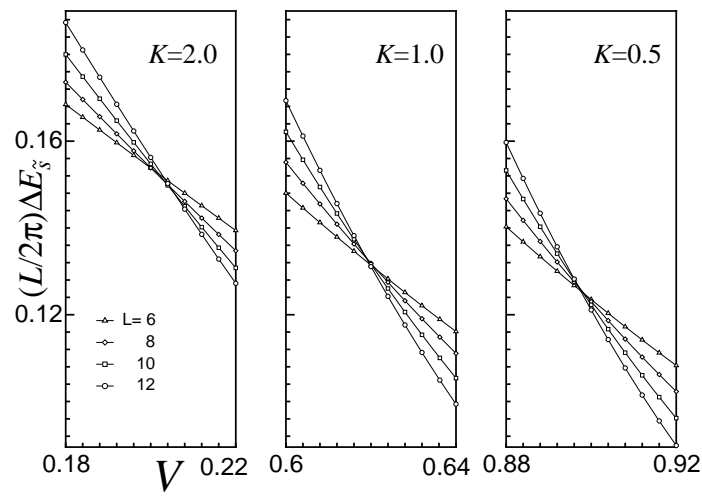

FIG. 1: The $V$ dependence of $(L / 2 \pi) \Delta E_{\tilde{s}}(K, V, L)$. From left to right, $K=2.0,1.0$ and 0.5 , respectively. The correspondence between marks and system sizes is given in the figure. The crossing points give $V_{3}(K, \bar{L})$.

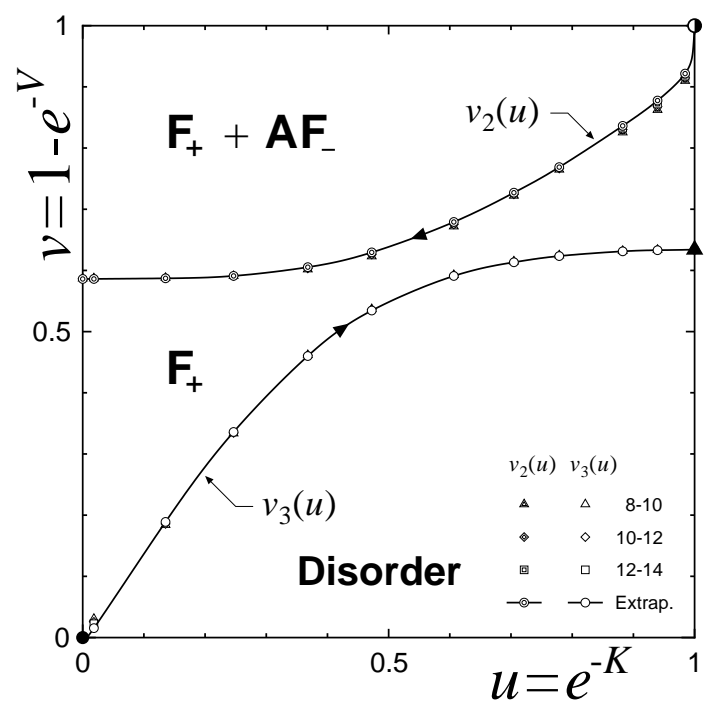

FIG. 2: The phase diagram of the Hamiltonian (2) in the space of the reduced parameters $(u, v)=\left(e^{-K}, 1-e^{-V}\right)$. Open circles (double circles) with a curve exhibit the phase boundary $v_{3}(u)\left[v_{2}(u)\right]$, which belongs to the three-state Potts (Ising) universality class (arrows show the directions of the RG-flows). The filled triangle on the $v$ biaxis denotes the $\mathrm{F}$ three-state Potts critical point $\left(1, v_{3}^{\mathrm{ex}}\right)$, and the filled (halffilled) circle corresponds to the ground state of the AF threestate Potts model on $\Lambda\left(\Lambda_{-}\right)$with the Gaussian criticality.

ter space within a unit square region. Then we draw the phase boundary line $v_{3}(u)$ corresponding to $V_{3}(K)$ (open circles with a fitting curve) in Fig. [2] The sizedependent data $V_{3}(K, \bar{L})$ are also given by other marks. From this figure, we can find the following: The extrapolated boundary line starts out of the filled circle $(u, v)=(0,0)$ linearly with the increase of $u$, which agrees with the crossover argument, i.e., $V_{3}(K) \propto e^{-K \phi_{3}}$ with $\phi_{3}=\left(2-x_{P}\right) /\left(2-x_{\epsilon}\right)=1[9,25]$. Then, $v_{3}(u)$ monotonically increases and finally terminates at the filled trian- gle $(u, v)=\left(1, v_{3}^{\mathrm{ex}}\right)$ with $v_{3}^{\mathrm{ex}}:=1-e^{-V_{3}^{\mathrm{ex}}}$, as expected. Consequently, the boundary line connects the Gaussian and the three-state Potts critical fixed points, which is in accord with Zamolodchikov's $c$-theorem [4].

The line $V_{3}(K)$ is now known to separate the disordered and the partially ordered phases, because the spin degrees on $\Lambda_{-}$are still disordered, at least, on the decoupling line, $K=0$ and $V>V_{3}^{\text {ex }}$. Therefore, another phase boundary to the ordered phase exists. To see this, let us investigate our model for large enough $V$. Since the F order is well established on $\Lambda_{+}$, we can approximately replace the spin degrees on $\Lambda_{+}$by a spontaneously favored value, e.g., $\tau$. Then, the subsystem on $\Lambda_{-}$is effectively described by the AF three-state Potts model under the uniform magnetic field; the effective Hamiltonian is

$$
\mathcal{H}_{-}(\tilde{K}, V)=\tilde{K} \sum_{j} \delta_{\sigma_{j}, \tau}+V \sum_{[j, k]} \delta_{\sigma_{j}, \sigma_{k}}
$$

$\left(j, k \in \Lambda_{-}\right)$. Again, the AF Potts criticality at $(K, V)=$ $(0, \infty)$ is perturbed by two relevant operators, but unlike in the case of Eq. (2) the $\mathbf{S}_{3}$ symmetry is absent, so that a different type of crossover may be expected. For large $\tilde{K}$, since $\sigma_{j}$ cannot take $\tau$, the subsystem is effectively described by the Ising model. Rácz and Vicsek investigated the Hamiltonian (5) by the use of the MC method, and obtained the boundary line [26], so that our next numerical task is to determine the second line $V_{2}(K)$ to complete the phase diagram of our Hamiltonian (2).

The relevant level in our PRG calculations may correspond to the magnetic-type excitation on the Ising critical fixed point $\Delta E_{\sigma}(K, V, L)$ with the scaling dimension $x_{\sigma}=\frac{1}{8}$, and it can be found in the above of the levels which are to be degenerate to the ground state. So, similarly to $V_{3}(K, \bar{L})$, we can calculate the size-dependent estimates of transition points $V_{2}(K, \bar{L})$ from the crossings of the scaled gaps $L \Delta E_{\sigma}(K, V, L)$. Then, extrapolating them to the thermodynamic limit by using the formula $V_{2}(K, L)-V_{2}(K) \propto L^{-\psi_{2}}$, with $\psi_{2}=3$ [23], we obtain $V_{2}(K)$ [the corresponding value $v_{2}(u)$ is exhibited by the double circles with a curve in Fig. 2. Although Eq. (5.) becomes exact in the limit $V \rightarrow \infty$, the obtained boundary line is qualitatively comparable to that in Ref. 26] also for finite couplings. Here, let us examine the limit $V_{2}(K \rightarrow \infty)$. Suppose that the complete $\mathrm{F}$ order is established on $\Lambda_{+}$, then the limit is given by the transition point of the Ising model on $\Lambda_{-}, V_{2}^{\text {ex }} \simeq 0.8814$ $\left(1-e^{-V_{2}^{\text {ex }}} \simeq 0.5858\right.$ in Fig. 2). However, the spin configurations which are neglected in this argument exist in the original Hamiltonian (2), so $V_{2}(\infty)$ may deviate from $V_{2}^{\text {ex }}$. Our numerical estimate, $V_{2}(\infty) \simeq 0.8820$, is quite close to $V_{2}^{\text {ex }}$. This implies that the contributions from the neglected spin configurations may be small around $V_{2}^{\text {ex }}$, and thus Eq. (5) is expected to provide a very good description even near $V_{2}^{\text {ex }}$.

Now, in order to evaluate the conformal anomaly numbers, we perform three point fitting on Eq. (4) based on 
TABLE II: The size-dependent estimates of the conformal anomaly numbers $c_{q}(L)$ at $(K, V)=\left(1, V_{q}(1)\right)(q=2,3)$. The fitting $c_{q}(L)=c_{q}(\infty)+b_{1} / L^{2}$ is performed using the data of $L=10$ and 12 .

\begin{tabular}{cccccc}
\hline \hline & $L=6$ & 8 & 10 & 12 & $\infty$ \\
\hline$c_{2}(L)$ & 0.5134201 & 0.4359578 & 0.4399469 & 0.4611012 & 0.5092 \\
$c_{3}(L)$ & 0.8012989 & 0.7682470 & 0.7714379 & 0.7815416 & 0.8045 \\
\hline \hline
\end{tabular}

$L-2, L$, and $L+2$, and estimate the size-dependent values $c_{q}(L)(q=2$ and 3$)$. As an example, we show them at intermediate couplings $(K, V)=\left(1, V_{q}(1)\right)$ in Table III Although nonmonotonic size dependences are visible, the extrapolated data agree well with the theoretical values, $\frac{1}{2}$ and $\frac{4}{5}$. For parameters close to the IR fixed points, i.e., $\left(K, V_{2}(K)\right)$ with $K \gg 1$ and $\left(K, V_{3}(K)\right)$ with $K \ll 1$, there are only small $L$ dependences, and it is easy to confirm the criticalities. However at $\left(K, V_{q}(K)\right)$ far away from the IR fixed points their precise estimations become difficult for the present system sizes.

Finally, we make a remark on the behavior around $(K, V)=(0, \infty)$ [i.e., the half-filled circle $(u, v)=(1,1)$ in Fig. 2. We have considered the model (2) possessing the global $\mathbf{S}_{3}$ symmetry. With the increase of $V, \mathbf{S}_{3}$ is broken at $V_{3}(K)$ and reduced to the $\mathbf{Z}_{2}$ symmetry with respect to the interchange of unfavored two spin states ("charge conjugation") for $V>V_{3}(K)$. While the lattice Hamiltonian (5) well describes the transition accompanied by the $\mathbf{Z}_{2}$ symmetry breaking [26, 27], corresponding field theory in the scaling limit is required to clarify, for instance, the phase boundary line around $(K, V)=(0, \infty)$. For this issue, it is plausible that the symmetry-breaking negative field $(\propto K)$ couples with the uniform magnetization $s_{j}$ and brings about a competition to the energy operator $\epsilon_{j}$ (a coupling $\propto e^{-V}$ ). By borrowing the sineGordon expression in Ref. [9], this competition may be formulated by the action preserving the sublattice symmetry and the charge conjugation but breaking the cyclic permutation symmetry as

$$
\mathcal{A}=\mathcal{A}_{c=1}-\int \mathrm{d}^{2} x\left[\mu \cos \beta \varphi+\bar{\mu} \cos \left(\frac{4 \pi}{\beta} \tilde{\varphi}\right)\right],
$$

where $\mathcal{A}_{c=1}$ is the free boson action, and $\tilde{\varphi}$ is the dual field of $\varphi$. The possibility that the dual sine-Gordon theory (6) describes the Ising criticality was mentioned by Delfino in relation to the Ashkin-Teller model [14, 28]. Further, note that the action (6) can become self-dual at $\beta^{2}=4 \pi$ which is known to exactly show the Ising criticality [17]. Based on this expression, we can predict a shape of the boundary line as follows: $K \propto e^{-V_{2}(K) \phi_{2}}$ with $\phi_{2}=\left(2-x_{s}\right) /\left(2-x_{\epsilon}\right)=\frac{8}{3}$. This seems to coincide with the rapid change of the boundary observed in Fig. 2 However, the precise estimation of $\phi_{2}$ is outside of the scope of our present research; the investigation to confirm this prediction is now in progress.
To summarize, we have investigated two crossovers of criticalities in the square-lattice three-state Potts antiferromagnet with staggered polarization field. On the basis of the dual sine-Gordon field theories, we have also given an argument on the criticalities and the shapes of the phase boundaries. For more detailed study, we are now performing the Monte Carlo simulation calculations to show the evidences for supporting the present result; we will give them in the forthcoming article.

Main computations were performed using the facilities of Yukawa Institute for Theoretical Physics.

[1] G.H. Wannier, Phys. Rev. 79, 357 (1950).

[2] R.M.F. Houtapple, Physica (Amsterdam) 16, 425 (1950); J. Stephenson, J. Math. Phys. (N.Y.) 5, 1009 (1964); ibid. 11, 420 (1970).

[3] For example, X. Qian, M. Wegewijs, and H.W.J. Blöte, Phys. Rev. E 69, 036127 (2004), and references therein.

[4] A.B. Zamolodchikov, Zh. Eksp. Teor. Fiz. 43, 565 (1986) [JETP Lett. 43, 730 (1986)].

[5] A. Lenard, cited in E.H. Lieb, Phys. Rev. 162, 162 (1967); E.H. Lieb, Phys. Rev. Lett. 18, 692 (1967).

[6] R.J. Baxter, J. Math. Phys. (N.Y.) 11, 3116 (1970); R.J. Baxter, Proc. Roy. Soc. London A383, 43 (1982).

[7] M.P.M. den Nijs, M.P. Nightingale, and M. Schick, Phys. Rev. B 26, 2490 (1982).

[8] J.K. Burton Jr. and C.L. Henley, J. Phys. A 30, 8385 (1997).

[9] G. Delfino, J. Phys. A 34, L311 (2001).

[10] J. Kolafa, J. Phys. A 17, L777 (1984); J.-S. Wang, R.H. Swendsen and R. Kotecký, Phys. Rev. Lett. 63, 109 (1989).

[11] J. Salas and A.D. Sokal, J. Stat. Phys. 92, 729 (1998).

[12] J.L. Cardy, J.L. Jacobsen, and A. Sokal, J. Stat. Phys. 105, 25 (2001).

[13] S.J. Ferreira and A.D. Sokal, J. Stat. Phys. 96, 461 (1999).

[14] G. Delfino, hep-th/0110181

[15] P. Lecheminant and E. Orignac, Phys. Rev. B 69, 174409 (2004).

[16] S.L.A. de Queiroz, Phys. Rev. E 65, 056104 (2002).

[17] P. Lecheminant, A.O. Gogolin, and A.A. Nersesyan, Nucl. Phys. B 639, 502 (2002).

[18] R.B. Potts, Proc. Cambridge. Philos. Soc. 48, 106 (1952).

[19] J.L. Cardy, J. Phys. A 17, L385 (1984).

[20] H.W. Blöte, J.L. Cardy and M.P. Nightingale, Phys. Rev. Lett. 56, 742 (1986); I. Affleck, ibid. 56, 746 (1986).

[21] For the 1D quantum systems; see H. Otsuka, Phys. Rev. B 66, 172411 (2002); H. Otsuka and M. Nakamura, Phys. Rev. B 70, 073105 (2004).

[22] M.P. Nightingale, Physica 83A, 561 (1976); H.H. Roomany and H.W. Wyld, Phys. Rev. D 21, 3341 (1980).

[23] B. Derrida and L. De. Seze, J. Phys. (Paris) 43, 475 (1982).

[24] B. Nienhuis, J. Phys. A 15, 199 (1982).

[25] J.L. Cardy, Scaling and Renormalization in Statistical Physics (Cambridge U.P., Cambridge, 1996).

[26] Z. Rácz and T. Vicsek, Phys. Rev. B 27, 2992 (1983).

[27] S.L.A. de Queiroz et al., Phys. Rev. E 59, 2772 (1999).

[28] G. Delfino, Phys. Lett. B 450, 196 (1999). 